\title{
Human albumin in the management of complications of liver cirrhosis
}

\author{
Mauro Bernardi ${ }^{2 *}$, Caterina Maggioli² and Giacomo Zaccherini² \\ This article is one of eleven reviews selected from the Annual Update in Intensive Care and Emergency Medicine 2012 (Springer Verlag, \\ DOI: 10.1007/978-3-642-25716-2) and co-published as a series in Critical Care. Other articles in the series can be found online at \\ http://ccforum.com/series/annualupdate2012. Further information about the Annual Update in Intensive Care and Emergency Medicine is \\ available from http://www.springer.com/series/8901.
}

\section{Introduction}

Human serum albumin is the most abundant plasma protein, representing about $50 \%$ of the total protein content $(3.5-5 \mathrm{~g} / \mathrm{l})$. Albumin is a protein of 585 amino acids and molecular weight $66 \mathrm{kDa}$ encoded by a gene on chromosome 4 and is exclusively synthesized by liver cells, which release it directly into the blood stream without storage. Under physiological conditions, only $20-30 \%$ of hepatocytes are committed to the production of 9$12 \mathrm{~g}$ of albumin per day; therefore, the liver has a large functional reserve, so that it can increase the synthesis of this protein by $3-4$ times, if necessary. The production of albumin is mainly regulated by the osmolarity and oncotic pressure of interstitial fluid in the liver extravascular space, but it is also induced by hormonal factors (insulin, cortisol and growth hormone) and inhibited by acute phase cytokines, such as interleukin (IL)-6 and tumor necrosis factor (TNF)- $\alpha$ [1].

Because serum albumin scarcely crosses the majority of capillaries, it remains in the blood stream and generates about $70 \%$ of the plasma oncotic pressure. This feature is two-thirds because of a direct osmotic effect and one third because of the Gibbs-Donnan effect, as a result of the strong negative charges attracting positively charged molecules into the intravascular compartment. Albumin is, therefore, a main modulator of fluid distribution throughout body compartments [1].

The capacity of albumin to expand the plasma volume represents the pathophysiological background for the use of human albumin in many clinical conditions; nevertheless, its administration is often inappropriate, largely

*Correspondence: mauro.bernardi@unibo.it

'Department of Anesthesia and Intensive Care, Cattinara Hospital, Strada di Fiume 447, 34149 Trieste, Italy

Full list of author information is available at the end of the article because of a common belief in its efficacy, whereas many indications are still under debate or have been disproved by evidence-based medicine. Indeed, the high cost, the theoretical risk of viral disease transmission, and the availability of cheaper alternatives should be carefully weighed when considering prescription of albumin [2]. At present, it is generally accepted that the administration of non-protein colloids and crystalloids represents the first-line treatment of resuscitation, and use of albumin in critically ill patients should be reserved for specific conditions, such as in patients with septic shock [3,4]. Albumin administration is not recommended to correct hypoalbuminemia per se (i.e., not associated with hypovolemia) or for nutritional intervention [2,5], but these indications are often disregarded in clinical practice. Albumin is also prescribed in certain specific conditions and diseases, such as kernicterus, plasmapheresis, and graft versus host disease [2], even though these indications are not supported by definite evidence.

Although the clinical use of albumin is mainly related to plasma volume expansion, albumin is more than a volume expander, having further biological properties: It binds and transports a variety of water-insoluble molecules, metals, and drugs, with implications for delivery and efficacy of drugs, including antibiotics, and for detoxification of endogenous and exogenous substances [5]. Furthermore, it constitutes the main circulating antioxidant in the body, being the major extracellular source of reduced sulfhydryl groups, potent scavengers of reactive oxygen species (ROS) (Figure 1) [6].

Among the 35 cysteine residues of albumin, 34 are employed for intramolecular disulfide bonds, whereas cysteine 34 (Cys34) remains free. ROS react primarily with Cys34, leading to reversible, highly unstable derivatives: The sulfenic and sulfinic acids. The subsequent, final product of the reaction is the sulfonic acid-albumin 


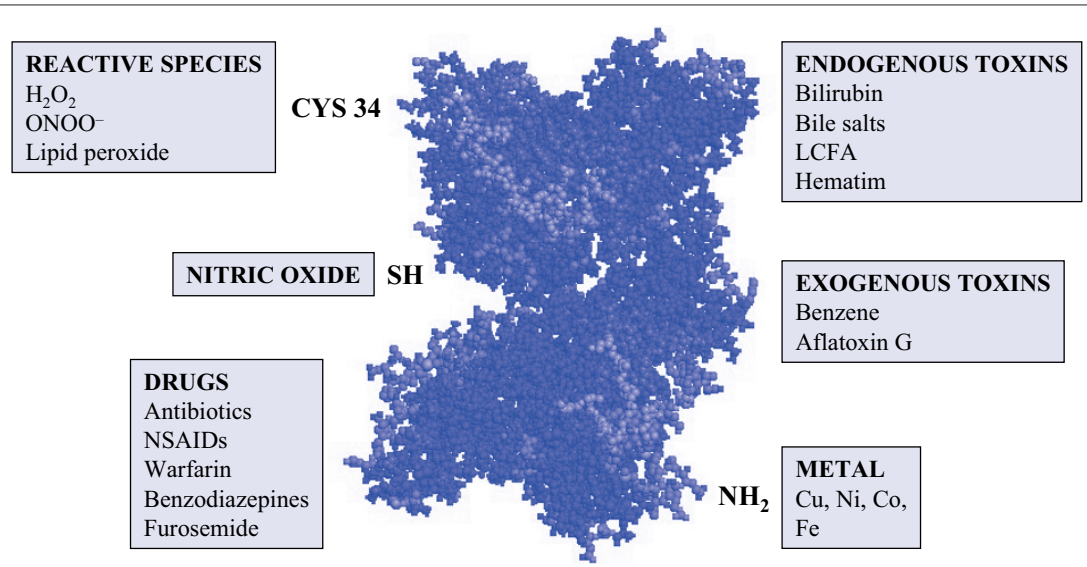

Figure 1. Non-oncotic properties of human albumin: binding, transport and detoxification capacities. $\mathrm{CYS}$, cysteine; $\mathrm{H}_{2} \mathrm{O}_{2}$, hydrogen peroxide; LCFA, long-chain fatty acids; NSAIDs, non-steroidal anti-inflammatory drugs; ONOO-, peroxynitrite.

complex. Oxidation alters the biological properties of albumin, which becomes more susceptible to proteinase digestion, undergoes faster degradation than the nonoxidized counterpart, and has reduced binding capacity to various substances, including bilirubin [6,7]. In healthy adults, about $70-80 \%$ of Cys 34 contains a free sulfhydryl group (human mercaptalbumin); about $25 \%$ forms a disulfide bond with small sulfhydryl compounds, such as cysteine, homocysteine or glutathione (human nonmercaptalbumin 1); and a small fraction is highly oxidized to sulfonic acid (human nonmercaptalbumin 2). In contrast, in chronic diseases, such as diabetes, renal failure, ischemic heart disease, and cancer, the oxidized form of albumin greatly increases, leading to an impairment of its biological activities [7]. Ischemia-modified albumin (IMA) has the capacity to bind cobalt and other metal ions, a property that contributes to the anti-oxidant activity of albumin by preventing these molecules from catalyzing pro-oxidative reactions. Increased IMA levels have been proposed as a mortality predictor in renal failure [8] and myocardial infarction [9].

\section{Human serum albumin and liver cirrhosis}

Patients with advanced cirrhosis almost always have hypoalbuminemia caused both by decreased synthesis by the hepatocytes and water and sodium retention that dilutes the content of albumin in the extracellular space. Other factors likely contribute to the development of hypoalbuminemia, including an increased transcapillary transport rate [10].

\section{Oncotic properties of albumin in cirrhosis}

The therapeutic use of albumin in hepatology dates back to the 1960s when it was thought that hypoalbuminemia had a prominent role in the pathogenesis of ascites, because of alteration of the balance between the forces of
Starling in the intrahepatic microcirculation. It was found that plasma albumin levels less than $3 \mathrm{~g} / \mathrm{l}$ were almost constantly associated with the presence of ascites, which did not develop with plasma levels greater than $4 \mathrm{~g} / \mathrm{l}$ [11]. Moreover, plasma oncotic pressure less than $20 \mathrm{mmHg}$ significantly increased the probability of developing ascites in the presence of portal hypertension [10]. These findings may support supplementation with exogenous albumin in patients with ascites and hypoalbuminemia. However, the net flow of fluid from the vascular compartment to the interstitium is regulated by its transcapillary gradient rather than by its intravascular concentration alone. When the gradient between plasma and ascitic fluid oncotic pressure was taken into account, no correlation was found with the rate of ascites formation [12], indicating that hypoalbuminemia simply reflects a deterioration in liver synthetic function without playing a major, direct role in the pathogenesis of ascites formation.

From the 1990s better understanding of the cardiovascular alterations in patients with advanced cirrhosis led to a critical review of the clinical use of albumin. These patients typically have a hyperdynamic circulatory syndrome, characterized by a decrease in peripheral vascular resistance and a compensatory increase in cardiac output, which manifest at the clinical level with arterial hypotension, tachycardia and hyperkinetic arterial pulses. The primary cause of the hyperdynamic circulatory syndrome is arterial vasodilation, mainly localized in the splanchnic circulatory area, of sufficient magnitude to reduce the effective blood volume (i.e., the blood volume in the heart, lungs and central arterial tree that is sensed by arterial receptors). Arterial vasodilation in cirrhosis mainly results from the increased production of vasoactive substances, such as nitric oxide (NO), carbon monoxide, and endocannabinoids, which induce vasodilation and hamper the vascular response to 
vasoconstrictors. The ensuing effective hypovolemia evokes the compensatory activation of neuro-humoral systems, including the renin-angiotensin-aldosterone axis, sympathetic nervous system, and arginine-vasopressin, able to promote vasoconstriction and renal retention of sodium and water. As a result, from a functional point of view, patients with advanced cirrhosis are hypovolemic and exhibit cardiovascular hyporeactivity, even though their cardiac output is usually significantly elevated [13]. However, a decrease in cardiac output leading to an exacerbation of effective hypovolemia is observed in patients with severe disease, suggesting clinically relevant cardiac dysfunction in the more advanced stages of cirrhosis [13]. In addition to ascites, these cardiovascular alterations represent the pathophysiological background of several severe complications of cirrhosis, such as hepatorenal syndrome (HRS), precipitated or not by spontaneous bacterial peritonitis (SBP), and postparacentesis circulatory dysfunction (PPCD), which all share acute exacerbation of effective hypovolemia as the pathophysiological momentum.

Based on this background, preservation of central blood volume represents a major aim in the management of patients with advanced cirrhosis. Several controlled and/or randomized studies have shown that albumin administration is effective to prevent circulatory dysfunction after large-volume paracentesis [14] and renal failure after SBP [15], and to treat HRS when given together with vasocontrictors $[16,17]$. Furthermore, it is currently believed that the capacity of albumin to expand the central blood volume in cirrhosis is superior to that of several other plasma-expanders [18]. In contrast, the chronic use of albumin to treat ascites is still debated, due to the lack of definitive scientific evidence supporting its clinical benefits. Thus, albumin is now given in patients with cirrhosis to expand effective volemia regardless of its plasma level.

\section{Prevention of post-paracentesis circulatory dysfunction}

Large-volume paracentesis is the treatment of choice for the management of patients with massive or refractory ascites. Although large-volume paracentesis is a safe procedure and the risk of local complications, such as hemorrhage or bowel perforation, is very low, it can favor the development of PPCD, which is characterized by the exacerbation of effective hypovolemia and is defined by an increase of more than $50 \%$ in the basal plasma renin activity 4-6 days after paracentesis. PPCD predisposes to rapid re-accumulation of ascites, hyponatremia, renal failure, and shortened survival [19].

The occurrence of PPCD is significantly reduced when large-volume paracentesis is combined with infusion of albumin ( $8 \mathrm{~g} / \mathrm{l}$ of ascites removed). Albumin was more effective than other plasma expanders (dextran-70 or polygeline) for the prevention of PPCD when more than $5 \mathrm{l}$ of ascites were removed; however, the incidence of PPCD was similar when less than $5 \mathrm{l}$ of ascites were tapped $[18,19]$. Moreover, use of albumin after largevolume paracentesis compared with alternative but cheaper plasma expanders appears to be more costeffective because albumin post-paracentesis is associated with fewer liver-related complications within the first month [20].

Despite these findings, the clinical value of albumin administration as an adjunct to large-volume paracentesis has been questioned because a survival benefit could not be shown in individual randomized trials. However, a meta-analysis that included all available randomized clinical trials comparing albumin with alternative treatments showed that albumin infusion significantly reduces the incidence of PPCD, hyponatremia and mortality, providing evidence-based support for the well-accepted clinical practice of infusing albumin as first choice in patients requiring large-volume paracentesis (Bernardi $\mathrm{M}$, unpublished results).

\section{Hepatorenal syndrome}

HRS is defined as the occurrence of renal failure in patients with advanced liver disease without another identifiable cause of renal failure, and is usually classified into two types according to the definition of the International Ascites Club [21]. Type 1 HRS is a rapidly progressive acute renal failure diagnosed when the serum creatinine increases more than $100 \%$ from baseline to a final level greater than $2.5 \mathrm{mg} / \mathrm{dl}$ within two weeks. It is usually precipitated by an acute insult often represented by a bacterial infection, although it may occur without any clear triggering event. Type 2 HRS is a relatively steady but moderate degree of functional renal failure with serum creatinine levels above $1.5 \mathrm{mg} / \mathrm{dl}$. It is usually associated with refractory ascites and often hyponatremia. Patients with type 2 HRS may eventually develop type 1 HRS either spontaneously or following a precipitating event, such as SBP.

HRS can be seen as the final event of the hemodynamic disturbances characterizing advanced cirrhosis. The development of HRS is related to an extreme reduction in the effective arterial blood volume combined with a decrease in the mean arterial pressure (MAP). This condition results from either a marked vasodilatation mainly in the splanchnic arterial bed or an impairment of cardiac function as a result of cirrhotic cardiomyopathy. Thus, the cardiac output in patients with HRS may be low or normal (infrequently high), but always insufficient for the patient's needs [21]. Effective hypovolemia produces a very intense compensatory activation of the sympathetic nervous and renin-angiotensin-aldosterone systems which causes renal vasoconstriction and a shift in the 


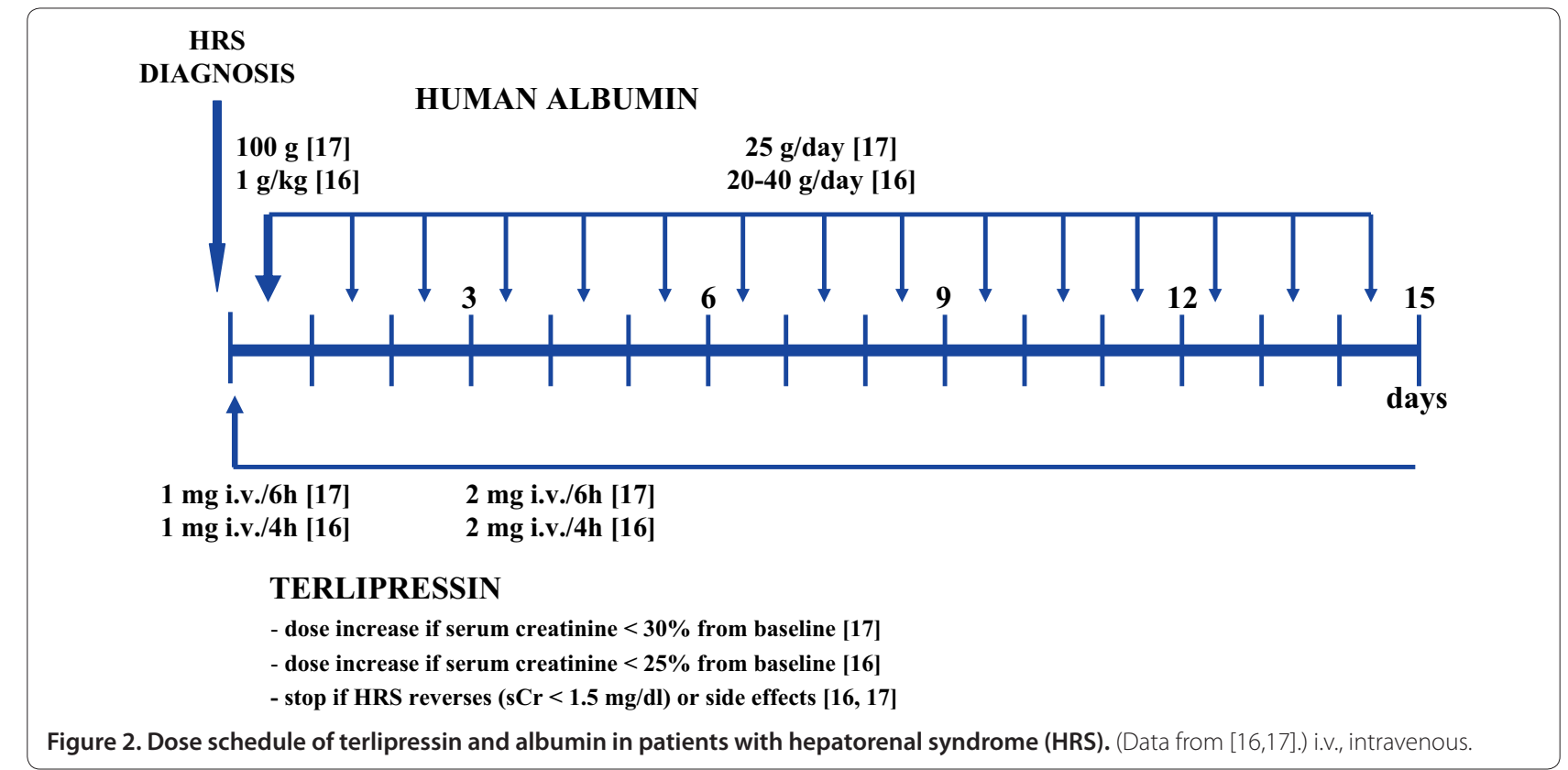

renal autoregulatory curve, rendering renal blood flow much more sensitive to the negative effects of low MAP [22].

The prognosis of type 1 HRS is dismal with almost $90 \%$ of patients dying without therapy within 2 weeks. In the last decade, combined therapy with vasoconstrictors and albumin has proved to be effective in reversing HRS in approximately half of the cases [22]. The rationale for this approach is to improve the markedly impaired circulatory function by causing vasoconstriction of the extremely dilated splanchnic vascular bed and by antagonizing effective hypovolemia so that MAP and renal perfusion can increase [22].

Terlipressin, a vasopressin analog, is the most studied vasoconstrictor and two multicenter randomized trials have demonstrated the efficacy of increasing doses of terlipressin in combination with albumin $(1 \mathrm{~g} / \mathrm{kg}$ or $100 \mathrm{~g}$ on day 1 followed by 20-40 g/day) (Figure 2) [16, 17]. Albumin administration appears necessary to improve the efficacy of the vasoconstrictor as the rate of HRS reversal is significantly lower with terlipressin alone compared to combination therapy [23]. The most frequent side effects of treatment are cardiovascular or ischemic complications, which have been reported in more than $10 \%$ of patients treated [24].

Vasoconstrictors other than vasopressin analogs that have been used in the management of type 1 HRS include norepinephrine and midodrine plus octreotide, both in combination with albumin. Although the number of patients enrolled in the studies evaluating these vasoconstrictors is quite small, their use appeared to improve renal function in a consistent number of patients with
HRS $[25,26]$. Finally, a systematic review of randomized studies using terlipressin as well as other vasoconstrictors, always in combination with albumin, has shown that treatment is associated with an improved short-term survival [24]. This result may be of paramount importance to keep patients waiting for liver transplantation alive until an organ becomes available [27].

\section{Prevention of hepatorenal syndrome after spontaneous bacterial peritonitis}

All patients with cirrhosis and ascites are at risk of developing a bacterial infection of the ascitic fluid, called SBP, which is diagnosed when the neutrophil count in ascitic fluid exceeds $250 / \mathrm{mm}^{3}$ as determined by microscopy [19]. SBP, even without septic shock, may precipitate circulatory dysfunction with severe liver failure, hepatic encephalopathy and type $1 \mathrm{HRS}$, causing the death of the patient in approximately $20 \%$ of cases despite infection resolution with antibiotic treatment [19].

Administration of albumin at a dose of $1.5 \mathrm{~g} / \mathrm{kg}$ body weight at diagnosis followed by $1 \mathrm{~g} / \mathrm{kg}$ on day 3 significantly decreased the incidence of type 1 HRS from $30 \%$ to $10 \%$ and reduced mortality from $29 \%$ to $10 \%$ compared with cefotaxime alone in a randomized, controlled study in patients with SBP (Figure 3) [15]. If the efficacy of albumin administration in patients with advanced disease, as wit nessed by a serum bilirubin greater than $4 \mathrm{mg} / \mathrm{dl}$ and a serum creatinine greater than $1 \mathrm{mg} / \mathrm{dl}$, appears to be undisputed, this is not the case for patients with moderate liver failure and without renal dysfunction at diagnosis as they show a very low incidence of HRS precipitated by SBP [15]. At the same time, 


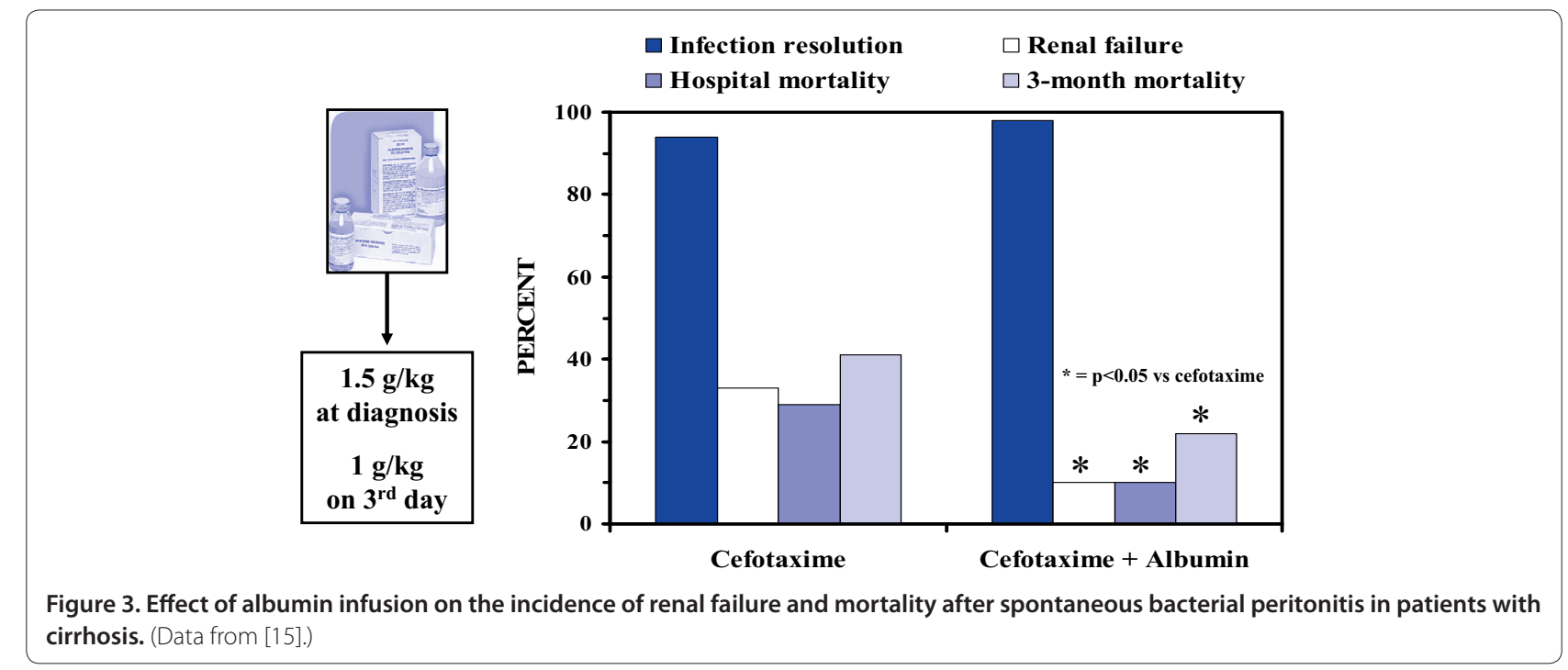

it is not established whether crystalloids or artificial colloids could replace albumin, although equivalent doses of hydroxyethyl starch (HES) have not been found to be as effective as albumin in preventing the circulatory dysfunction caused by SBP [28]. Interestingly, albumin infusion was able to prevent the increase in nitrate levels and to reduce the elevated plasma levels of Von Willebrand-related antigen observed in patients with SBP treated with HES [29]. This finding suggests an effect of albumin on endothelial function that appears to be related to the non-oncotic properties of this molecule rather than to its activity as plasma-expander.

\section{Ascites}

The chronic use of albumin to treat ascites is still debated, due to the lack of definitive scientific evidence supporting its clinical benefits. When human albumin became available, studies performed several decades ago failed to show clear usefulness of this substance in relieving ascites and preventing its recurrence; however, these investigations were uncontrolled and/or included a small number of patients [30]. Thus, treatment of cirrhotic ascites with diuretics and albumin has been practiced on anecdote and experience for many years. More than a decade ago, a controlled clinical trial in hospitalized patients with cirrhosis and ascites randomized to receive diuretics associated or not with low-doses of albumin (12.5 g/day) showed that treatment with diuretics plus albumin was overall more effective than diuretics alone in determining the disappearance of ascites and reducing the length of the hospital stay [31]. However, this result was only achieved in patients who received the initial dose of diuretics, whereas the difference in the treatment response was not statistically significant in those patients who required higher doses of diuretics or were refractory to diuretic treatment. Albumin administration at home also reduced the rate of ascites recurrence and readmission to the hospital due to ascites, but its prescription was very expensive and no effect on survival was observed [31]. More recently, the same research group showed, in an unblinded randomized trial, that longterm albumin administration is also able to increase patient survival and to reduce the risk of ascites recurrence [32].

No other controlled clinical trials have so far been performed to evaluate the effectiveness of prolonged albumin administration in the treatment of cirrhosis and ascites. Thus, the absence of confirmatory multicenter randomized studies, together with the high cost of human albumin, explains why albumin infusion is not usually included among the therapeutic options for difficult-to-treat ascites. A multicenter randomized clinical trial (NCT 01288794, www.clinicaltrials.gov) planning to enroll more than 400 patients is ongoing in Italy to cover this lack of information.

\section{Non-oncotic properties of albumin in cirrhosis}

In recent years, scientific interest into the non-oncotic properties of human serum albumin has progressively increased in the field of hepatology. Initial findings showed that patients with chronic liver disease have decreased amounts of circulating human nonmercaptalbumin (the 'good guy') and elevated levels of the oxidized form human nonmercaptalbumin 26 (the 'bad guy') $[33,34]$. More recently, binding, transport, and detoxification capacities of albumin have been found to be severely impaired in cirrhotic patients, particularly in those with acute-on-chronic liver failure precipitated by bacterial infections [35]. As a result, endogenous and exogenous substances that are normally bound to 
albumin are free to react arbitrarily. The reduced detoxification capacity of albumin is especially important, as it suggests that the molecule cannot bind and remove waste products. Furthermore, IMA is significantly increased, suggesting an impaired metal chelation capacity that contributes to generate an environment of sustained oxidative stress by favoring radical formation through Fenton chemistry processes. Most important, the loss of albumin function and the increase in the IMA level were associated with poor survival $[8,9,35]$.

From a clinical context, the non-oncotic properties of albumin have so far been exploited in extracorporeal albumin dialysis systems in patients with acute or acuteon-chronic liver failure. The two commercially available detoxification systems are the "Molecular Adsorbent Recirculating System" (MARS) and the "fractionated plasma separation and adsorption" (Prometheus), which are based on the association of a conventional dialysis membrane with a second dialysis circuit filled with a circulating 20\% albumin solution in the case of MARS or with a second filter containing albumin in the case of Prometheus. In addition to the positive consequences associated with the removal of toxic molecules, early studies of these systems showed a favorable impact on systemic hemodynamics likely due to the purification of substances involved in the pathogenesis of the hyperdynamic circulatory syndrome, such as NO, and the proinflammatory cytokines, TNF- $\alpha$ and IL-6 [36,37]. Such systems, currently available only in specialized and transplant centers, have a potential therapeutic role in the following medical conditions: Acute fulminant liver failure, acute liver failure on chronic liver disease, primary dysfunction of the transplanted liver, liver failure after resection and intractable pruritus [37]. It has to be stressed, however, that the results of the first large-scale controlled trials that have employed either MARS or Prometheus in patients with cirrhosis and acute-onchronic liver failure did not show a significant impact on major outcomes, such as survival [38,39]. Likely, subgroups of patients who would actually benefit from these detoxification systems need to be identified, as suggested by the survival benefit observed in patients with severe cirrhosis (model for end-stage liver disease [MELD] score > 30) and HRS type 1 treated with the Prometheus system [39].

\section{Conclusion}

The use of human albumin in the setting of liver cirrhosis is supported by evidence arising from prospective randomized trials and meta-analyses. Albumin administration is, therefore widely accepted and recommended by current international guidelines for the prevention of PPCD and acute renal failure in patients with SBP, and the treatment of HRS as an adjunct to vasoconstrictors.
All these complications share the common pathophysiological background of reduced effective volemia, mainly because of peripheral arterial vasodilation, and the rationale underlying the use of albumin is related to its activity as a plasma expander. However, it is likely that the beneficial effects of albumin are also linked to its non-oncotic properties, including binding capacity, antioxidant activity, and effects on capillary integrity. These effects represent the main fields of current and future research, from which will likely arise further and more appropriate indications for albumin administration in patients with liver disease.

\section{Abbreviations}

CYS, cysteine; Cys34, cysteine 34; $\mathrm{H}_{2} \mathrm{O}_{2}$, hydrogen peroxide; $\mathrm{HES}$, hydroxyethyl starch; HRS, hepatorenal syndrome; i.v., intravenous; IL, interleukin; IMA, Ischemia-modified albumin; LCFA, long-chain fatty acids; MAP, mean arterial pressure; MARS, Molecular Adsorbent Recirculating System; MELD, model for end-stage liver disease; NO, nitric oxide; NSAIDs, non-steroidal antiinflammatory drugs; ONOO, peroxynitrite; PPCD, post-paracentesis circulatory dysfunction; ROS, reactive oxygen species; SBP, spontaneous bacterial peritonitis; TNF, tumor necrosis factor.

\section{Competing interests}

The authors declare that there are no competing interests.

\section{Author details}

'Department of Anesthesia and Intensive Care, Cattinara Hospital, Strada di Fiume 447, 34149 Trieste, Italy. ${ }^{2}$ Department of Clinical Medicine, S. OrsolaMalpighi Polyclinic, Via Albertoni 15, 40138 Bologna, Italy.

Published: 20 March 2012

\section{References}

1. Quinlan GJ, Martin GS, Evans TW: Albumin: biochemical properties and therapeutic potential. Hepatology 2005, 41:1211-1219.

2. Mirici-Cappa F, Caraceni P, Domenicali M, et al:. How albumin administration for cirrhosis impacts on hospital albumin consumption and expenditure. World J Gastroenterol 2011, 17:3479-3486.

3. Finfer S, Bellomo R, Boyce N, French J, Myburgh J, Norton R; SAFE Study Investigators: A comparison of albumin and saline for fluid resuscitation in the intensive care unit. N Engl J Med 2004, 350:2247-2256.

4. Delaney AP, Dan A, McCaffrey J, Finfer S: The role of albumin as a resuscitation fluid for patients with sepsis: a systematic review and metaanalysis. Crit Care Med 2011, 39:386-391.

5. Vincent JL: Relevance of albumin in modern critical care medicine. Best Pract Res Clin Anaesthesiol 2009, 23:183-191.

6. Evans TW: Review article: albumin as a drug. Biological effects of albumin unrelated to oncotic pressure. Aliment Pharmacol Ther 2002, 16:56-S11.

7. Oettl K, Stauber RE: Physiological and pathological changes in the redox state of human serum albumin critically influence its binding properties. BrJ Pharmacol 2007, 151:580-590.

8. Sharma R, Gaze DC, Pellerin D, et al: Ischemia-modified albumin predicts mortality in ESRD. Am J Kidney Dis 2006, 47:493-502.

9. Van Belle E, Dallongeville J, Vicaut E, Degrandsart A, Baulac C, Montalescot G; OPERA Investigators: Ischemia-modified albumin levels predict long-term outcome in patients with acute myocardial infarction. The French Nationwide OPERA study. Am Heart J 2010, 159:570-576.

10. Henricksen JH, Siemssen O, Krintel JJ, Malchow-Møller A, Bendtsen F, RingLarsen H: Dynamics of albumin in plasma and acitic fluid in patients with cirrhosis. J Hepatol 2001, 34:53-60.

11. Wood LJ, Colman J, Dudley FJ: The relationship between portal pressure and plasma albumin in the development of cirrhotic ascites. J Gastroenterol Hepatol 1987, 2:525-531.

12. Rector WG Jr, Ibarra F, Openshaw K, Hoefs JC: Ascites kinetics in cirrhosis: relationship to plasma-ascites hydrostatic-oncotic balance and intensity of renal sodium retention. J Lab Clin Med 1986, 107:412-419. 
13. Møller S, Henriksen JH: Cardiovascular complications of cirrhosis. Gut 2008 57:268-278

14. Ginés P, Titó L, Arroyo V, et al:: Randomized comparative study of therapeutic paracentesis with and without intravenous albumin in cirrhosis. Gastroenterology 1998, 94:1493-1502.

15. Sort $P$, Navasa $M$, Arroyo $V$, et al:: Effect of intravenous albumin on renal impairment and mortality in patients with cirrhosis and spontaneous bacterial peritonitis. N Engl J Med 1999, 341:403-409.

16. Martín-Llahí M, Pépin MN, Guevara M, et al:: Terlipressin and albumin vs albumin in patients with cirrhosis and hepatorenal syndrome: a randomized study. Gastroenterology 2008, 134:1352-1359.

17. Sanyal AJ, Boyer T, Garcia-Tsao G, et al:: A randomized, prospective, doubleblind, placebo-controlled trial of terlipressin for type 1 hepatorenal syndrome. Gastroenterology 134:1360-1368.

18. Ginès A, Fernandez-Esparrach G, Monescillo A, et al:: Randomized controlled trial comparing albumin, dextran-70 and polygelin in cirrhotic patients with ascites treated by paracentesis. Gastroenterology 111:1002-1010.

19. European Association for the Study of the Liver: EASL clinical practice guidelines on the management of ascites, spontaneous bacterial peritonitis, and hepatorenal syndrome in cirrhosis. J Hepatol 2010, 53:397-417

20. Moreau R, Valla DC, Durand-Zaleski I, et al: Comparison of outcome in patients with cirrhosis and ascites following treatment with albumin or a synthetic colloid: a randomised controlled pilot trail. Liver Int 2006, 26:46-54

21. Salerno F, Gerbes $A$, Ginès $P$, Wong $F$, Arroyo V: Diagnosis, prevention and treatment of hepatorenal syndrome in cirrhosis. Gut 2007, 56:1310-1318.

22. Ginès P, Schrier RW: Renal failure in cirrhosis. N Engl J Med 2009, 361:1279-1290

23. Ortega R, Gines P, Uriz J, et al:: Terlipressin therapy with and without albumin for patients with hepatorenal syndrome: results of prospective, non-randomized study. Hepatology 2002, 36:941-948.

24. Gluud LL, Christensen K, Christensen E, Krag A: Systematic review of randomized trials on vasoconstrictor drugs for hepatorenal syndrome. Hepatology 2010, 51:576-584.

25. Angeli P, Volpin R, Gerunda G, et al:: Reversal of type 1 hepatorenal syndrome (HRS) with the combined administration of midodrine and octreotide. Hepatology 1999, 29:1690-1697.

26. Alessandria C, Ottobrelli A, Debernardi-Venon W, et al:: Noradrenalin vs terlipressin in patients with hepatorenal syndrome: a prospective, randomized, unblinded, pilot study. J Hepatol 2007, 47:499-505.

27. Caraceni P, Santi L, Mirici F, et al:: Long-term treatment of hepatorenal syndrome as a bridge to liver transplantation. Dig Liver Dis 2011, $43: 242-245$
28. Sigal SH, Stanca CM, Fernandez J, Arroyo V, Navasa M: Restricted use of albumin for spontaneous bacterial peritonitis. Gut 2007, 56:597-599.

29. Fernández J, Monteagudo J, Bargallo X, et al:: A randomized unblinded pilot study comparing albumin versus hydroxyethyl starch in spontaneous bacterial peritonitis. Hepatology 2005, 42:627-634.

30. Clermont RJ, Vlahevic ZR, Chalmers TC, Adham NF, Curtis GW, Morrosin RS Intravenous therapy of massive ascites in patients with cirrhosis. Longterm effects on survival and frequency of renal failure. Gastroenterology 1967, 53:220-228

31. Gentilini P, Casini-Raggi V, Di Fiore G, et al:: Albumin improves the response to diuretics in patients with cirrhosis and ascites: results of a randomized, controlled trial. J Hepatol 1999, 30:639-645.

32. Romanelli RG, La Villa G, Barletta G, et al.: Long-term albumin infusion improves survival in patients with cirrhosis and ascites: an unblinded randomized trial. World J Gastroenterol 2006, 12:1403-1407.

33. Watanabe A, Matsuzaki S, Moriwaki H, Suzuki K, Nishiguchi S: Problems in serum albumin measurement and clinical significance of albumin microheterogeneity in cirrhotics. Nutrition 2004, 20:351-357.

34. Oettl K, Stadlbauer $V$, Petter F, et al.: Oxidative damage of albumin in advanced liver disease. Biochim Biophys Acta 2008, 1782:469-473.

35. Jalan R, Schnurr K, Mookerjee RP, et al: Alterations in the functional capacity of albumin in patients with decompensated cirrhosis is associated with increased mortality. Hepatology 2009, 50:555-564.

36. Oettl K, Stadlbauer V, Krisper P, Stauber RE: Effect of extracorporeal liver support by molecular adsorbents recirculating system and Prometheus on redox state of albumin in acute-on-chronic liver failure. Ther Apher Dial 2009, 13:431-436.

37. Karvellas CJ, Gibney N, Kutsogiannis D, Wendon J, Bain VG: Bench-to-bedside review: current evidence for extracorporeal albumin dialysis systems in liver failure. Crit Care 2007, 11:215

38. Bañares R, Nevens F, Larsen FS, Jalan R, Albillos A, Dollinger M; Relief Study Group: Extracorporeal liver support with the molecular adsorbent recirculating system (MARS) in patients with acute-on-chronic liver failure (AOCLF). The RELIEF Trial. J Hepatol 2010, 52:S459-S460.

39. Rifai K, Kribben A, Gerken G, et al.: Extracorporeal liver support by fractionated plasma separation and adsorption (PROMETHEUS) in patients with acute-on-chronic liver failure (HELIOS Study): a prospective randomized controlled multicenter study. J Hepatol 2010, 52:S3.

doi:10.1186/cc11218

Cite this article as: Bernardi $M$, et al:. Human albumin in the management of complications of liver cirrhosis. Critical Care 2012, 16:211. 